\title{
CONSERVATION LAWS AND CONSERVED QUANTITIES FOR LAMINAR RADIAL JETS WITH SWIRL
}

\author{
R. $\mathrm{Naz}^{1}$, I. Naeem ${ }^{2}$ and F.M. Mahomed ${ }^{3}$ \\ ${ }^{1}$ Centre for Mathematics and Statistical Sciences Lahore School of Economics, \\ Lahore 53200, Pakistan. \\ ${ }^{2}$ Department of Mathematics, School of Science and Engineering, \\ LUMS, Lahore Cantt 54792, Pakistan. \\ ${ }^{3}$ School of Computational and Applied Mathematics, Centre for Differential \\ Equations, Continuum Mechanics and Applications, University of the \\ Witwatersrand, Wits 2050, South Africa. \\ rehananaz_qau@yahoo.com, inqau@yahoo.com, Fazal.Mahomed@wits.ac.za
}

\begin{abstract}
Conserved quantities play a central role in the solution of jet flow problems. A systematic way of deriving conserved quantities for the radial jets with swirl is presented. The multiplier approach is used to derive the conservation laws for the system of three boundary layer equations for the velocity components and for the system of two partial differential equations for the stream function. When the swirl is zero or at a large distance from the orifice (at infinity), the boundary layer equations for the radial jets with swirl reduce to those of the purely radial jets. The conserved quantities for the radial liquid, free and wall jets with swirl are derived by integrating the conservation laws across the jets.
\end{abstract}

Keywords- Radial jets with swirl, conservation laws, conserved quantity.

\section{INTRODUCTION}

It is of great interest to study the three dimensional boundary layer flows, for example the radial jets with swirl due to their extensive range of applications.

The concept of two-dimensional boundary layer flow of the purely radial liquid jet was introduced by Watson [1]. The two-dimensional boundary layer flow of the purely radial free jet was first considered by Schwarz [2]. Glauert in [3] studied the two-dimensional boundary layer flow of the purely radial wall jet. Later, Riley in [4] also discussed the two-dimensional boundary layer flow of purely radial liquid, free and wall jets. In all these jet flow problems, the boundary conditions are homogeneous and therefore the unknown exponent in the similarity solution cannot be obtained from the boundary conditions. By integrating Prandtl's momentum boundary layer equation across the jet and using the boundary conditions and the 
continuity equation, a condition, known as the conserved quantity, was derived. Riley [5] extended the problem of the purely radial jets to the radial jets with swirl. Later, O'Nan and Schwarz [6] also studied the problem of the radial free jet with swirl. The governing equations consist of a continuity equation and two momentum equations in the radial and swirl directions respectively. This problem consists of homogeneous boundary conditions and thus also requires conserved quantities. The conserved quantities for the radial jets with swirl were established using the same idea as was used in $[1,2,3,4]$ to construct the conserved quantities for the purely radial jets. By integrating the momentum equation in the swirl direction and using the boundary conditions and the continuity equation, one conserved quantity for the radial free jet with swirl and one for the radial wall jet with swirl were derived. The second conserved quantity for the radial free and wall jets with swirl were established by integrating the momentum equation in the radial direction and requiring that swirl is zero at $x=\infty$. The only conserved quantity for the radial liquid jet with swirl [5] was obtained from a physical argument based on conservation of mass flux in an incompressible fluid and is the same as for the purely radial liquid jet.

The conserved quantity for each problem has in general physical significance. Riley [5] established the conserved quantities for the radial liquid, free and wall jets with swirl. The only conserved quantity for the radial liquid jet with swirl is total mass flux per radian which is constant. One of the conserved quantities for the radial free jet with swirl is the radial flux of angular momentum about $x=0$. The second conserved quantity for the radial free jet with swirl is the radial flux of momentum and is invariant only when the swirl is zero or at large distances from the orifice. For a radial wall jet with swirl one conserved quantity is the flux of exterior momentum flux and is constant only when the swirl is zero or at large distances from the orifice. There is no obvious physical interpretation of the other conserved quantity for the radial wall jet with swirl.

In this paper, we construct the conserved quantities for the radial liquid, free and wall jets with swirl by the method recently introduced by Naz et al [7]. We use the multiplier approach introduced by Steudel [8] (see also $[9,10]$ ) to derive the conservation laws for the system of three partial differential equations for the velocity components as well as for the system of two partial differential equations for the stream function. The radial jets with swirl behave as purely radial jets when the swirl is zero or at large distances from the orifice as $x$ becomes large the swirl component will tend to zero more rapidly than the radial velocity component. Then the boundary layer equations for radial jets with swirl reduce to that of purely radial jets. The conservation laws for the purely radial jets were derived in [7] with the 
help of the multiplier approach. The conserved quantities for the radial, liquid, free and wall jets with swirl are derived with the help of conservation laws. The radial jets (liquid, free and wall) with swirl satisfy the same partial differential equations but the boundary conditions for each jet are different. The derivation of conservation laws depends only on the partial differential equations but the derivation of the physical conserved quantities depends also on the boundary conditions. The boundary conditions therefore determine which conserved vector is associated with which jet.

\section{CONSERVATION LAWS FOR RADIAL LAMINAR JET}

We consider the radial liquid jet, free jet and wall jet with swirl.

The radial liquid jet with swirl is formed when a circular jet of liquid, with an axially symmetrical swirling component of velocity, strikes a plane boundary normally and spreads over it. The radial free jet with swirl is formed when two circular jets, each with an axially-symmetrical swirling component of velocity, impinge on one another. When a circular jet, having axially symmetric swirling component of velocity, strikes a plane surface normally which is surrounded by the same fluid as the jet and spreads out radially over it then a radial wall jet with swirl is formed. All these jets were described by Riley [5].

The fluid in the jets is viscous and incompressible. In the free jet and wall jet the surrounding fluid consists of the same fluid as in the jet but for the liquid jet the surrounding fluid is a gas. The cylindrical polar coordinates $(x, \theta, y)$ are used with $x$ as radial coordinate and $x=0$ is the axis of symmetry. All fluid variables are independent of $\theta$. For the liquid jet the solid boundary is at $y=0$ and the free surface is at $y=\phi(x)$. The wall jet has a solid boundary at $y=0$ and the free jet is symmetrical about $y=0$. For all three jets an axially symmetrical swirling component of velocity is considered.

The boundary layer equations governing the flow in a radial laminar jet with swirl, in the absence of pressure gradient, are

$$
\begin{aligned}
& u u_{x}+v u_{y}-\frac{w^{2}}{x}=\nu u_{y y}, \\
& u w_{x}+v w_{y}+\frac{u w}{x}=\nu w_{y y}, \\
& (x u)_{x}+(x v)_{y}=0,
\end{aligned}
$$

where $u(x, y)$ and $v(x, y)$ are the velocity components in $x$ and $y$ directions respectively, $w(x, y)$ is an axially-symmetrical swirling component of velocity and $\nu$ is the 
kinematic viscosity of the fluid. Equations (1) and (2) are the momentum equations in the radial and $\theta$ directions respectively and (3) is the continuity equation. The boundary-layer equations (1)-(3) have been derived with the assumption that the swirl velocity $w$ and radial velocity $u$ are of same order of magnitude whereas the transverse velocity $v$ is of lower order (see [5]).

The stream function $\psi(x, y)$ satisfies [11]

$$
x u=\psi_{y}, x v=-\psi_{x} .
$$

The radial free jet with swirl is symmetrical about the plane $y=0$, therefore $v(x, 0)=0$. For the liquid jet and the wall jet there is no suction or blowing of fluid at the solid boundary $y=0$ and thus $v(x, 0)=0$. Therefore $\psi_{x}(x, 0)=0$ for all three jets and thus $\psi(x, 0)$ is a constant. We choose the constant as zero and hence

$$
\psi(x, 0)=0
$$

Let $O$ be any reference point on the line $y=0$ in the axial plane $\theta=\theta_{o}$, then the stream function at $P\left(x, \theta_{o}, y\right)$ is (see [11])

$$
\psi(x, y)=\int_{O}^{P} x[u(x, y) d y-v(x, y) d x] .
$$

Integrating (6) with respect to $y$ from $y=0$ to $y=\infty$ along a straight line with $x$ kept fixed yields

$$
\psi(x, \infty)=\int_{0}^{\infty} x u(x, y) d y .
$$

Now, $\psi(x, \infty)$ is finite only if we assume $u(x, y) \rightarrow 0$ sufficiently rapidly as $y \rightarrow \infty$.

Equation (3) is identically satisfied and equations (1) and (2) yield

$$
\frac{1}{x} \psi_{y} \psi_{x y}-\frac{1}{x^{2}} \psi_{y}^{2}-\frac{1}{x} \psi_{x} \psi_{y y}-\nu \psi_{y y y}-w^{2}=0
$$

and

$$
\frac{1}{x} \psi_{y} w_{x}-\frac{1}{x} \psi_{x} w_{y}+\frac{1}{x^{2}} \psi_{y} w-\nu w_{y y}=0
$$

We will derive the conservation laws for the system (1)-(3) and also for the system (8)-(9) for the stream function, using the multiplier approach [8, 9]. 


\subsection{Conservation laws for system of equations for velocity components}

Consider the multipliers of the form

$$
\Lambda_{1}=\Lambda_{1}(x, y, u, v, w), \Lambda_{2}=\Lambda_{2}(x, y, u, v, w) \operatorname{and} \Lambda_{3}=\Lambda_{3}(x, y, u, v, w)
$$

for the system of equations (1)-(3), such that

$$
\begin{array}{r}
\Lambda_{1}\left(u u_{x}+v u_{y}-\nu u_{y y}-\frac{w^{2}}{x}\right)+\Lambda_{2}\left(u w_{x}+v w_{y}+\frac{u w}{x}-\nu w_{y y}\right) \\
+\Lambda_{3}\left(u+x u_{x}+x v_{y}\right)=D_{x} T^{1}+D_{y} T^{2},
\end{array}
$$

for all functions $u(x, y), v(x, y)$ and $w(x, y)$ and not only for the solutions of system. In (10), $D_{x}$ and $D_{y}$ are total derivative operators defined by

$$
\begin{aligned}
& D_{x}=\frac{\partial}{\partial x}+u_{x} \frac{\partial}{\partial u}+v_{x} \frac{\partial}{\partial v}+w_{x} \frac{\partial}{\partial w}+u_{x x} \frac{\partial}{\partial u_{x}}+v_{x x} \frac{\partial}{\partial v_{x}}+w_{x x} \frac{\partial}{\partial w_{x}} \\
& +u_{x y} \frac{\partial}{\partial u_{y}}+v_{x y} \frac{\partial}{\partial v_{y}}+w_{x y} \frac{\partial}{\partial w_{y}}+\cdots, \\
& D_{y}=\frac{\partial}{\partial y}+u_{y} \frac{\partial}{\partial u}+v_{y} \frac{\partial}{\partial v}+w_{y} \frac{\partial}{\partial w}+u_{y y} \frac{\partial}{\partial u_{y}}+v_{y y} \frac{\partial}{\partial v_{y}}+w_{y y} \frac{\partial}{\partial w_{y}} \\
& +u_{y x} \frac{\partial}{\partial u_{x}}+v_{y x} \frac{\partial}{\partial v_{x}}+w_{y x} \frac{\partial}{\partial w_{x}}+\cdots .
\end{aligned}
$$

The determining equations for the multipliers $\Lambda_{1}, \Lambda_{2}$ and $\Lambda_{3}$ are

$$
\begin{aligned}
& E_{u}\left[\Lambda_{1}\left(u u_{x}+v u_{y}-\nu u_{y y}-\frac{w^{2}}{x}\right)+\Lambda_{2}\left(u w_{x}+v w_{y}+\frac{u w}{x}-\nu w_{y y}\right)\right. \\
& \left.+\Lambda_{3}\left(u+x u_{x}+x v_{y}\right)\right]=0 \\
& E_{v}\left[\Lambda_{1}\left(u u_{x}+v u_{y}-\nu u_{y y}-\frac{w^{2}}{x}\right)+\Lambda_{2}\left(u w_{x}+v w_{y}+\frac{u w}{x}-\nu w_{y y}\right)\right. \\
& \left.+\Lambda_{3}\left(u+x u_{x}+x v_{y}\right)\right]=0, \\
& E_{w}\left[\Lambda_{1}\left(u u_{x}+v u_{y}-\nu u_{y y}-\frac{w^{2}}{x}\right)+\Lambda_{2}\left(u w_{x}+v w_{y}+\frac{u w}{x}-\nu w_{y y}\right)\right. \\
& \left.+\Lambda_{3}\left(u+x u_{x}+x v_{y}\right)\right]=0
\end{aligned}
$$

where $E_{u}, E_{v}$ and $E_{w}$ are the standard Euler operators which annihilate divergence expressions:

$$
E_{u}=\frac{\partial}{\partial u}-D_{x} \frac{\partial}{\partial u_{x}}-D_{y} \frac{\partial}{\partial u_{y}}+D_{x}^{2} \frac{\partial}{\partial u_{x x}}+D_{x} D_{y} \frac{\partial}{\partial u_{x y}}+D_{y}^{2} \frac{\partial}{\partial u_{y y}}-\cdots
$$




$$
\begin{aligned}
& E_{v}=\frac{\partial}{\partial v}-D_{x} \frac{\partial}{\partial v_{x}}-D_{y} \frac{\partial}{\partial v_{y}}+D_{x}^{2} \frac{\partial}{\partial v_{x x}}+D_{x} D_{y} \frac{\partial}{\partial v_{x y}}+D_{y}^{2} \frac{\partial}{\partial v_{y y}}-\cdots \\
& E_{w}=\frac{\partial}{\partial w}-D_{x} \frac{\partial}{\partial w_{x}}-D_{y} \frac{\partial}{\partial w_{y}}+D_{x}^{2} \frac{\partial}{\partial w_{x x}}+D_{x} D_{y} \frac{\partial}{\partial w_{x y}}+D_{y}^{2} \frac{\partial}{\partial w_{y y}}-\cdots
\end{aligned}
$$

The expansion of equations (13)-(15) yields

$$
\begin{aligned}
& \Lambda_{1 u}\left(u u_{x}+v u_{y}-\nu u_{y y}-\frac{w^{2}}{x}\right)+\Lambda_{2 u}\left(u w_{x}+v w_{y}+\frac{u w}{x}-\nu w_{y y}\right)+\Lambda_{3 u}\left(u+x u_{x}+x v_{y}\right) \\
& +\Lambda_{1} u_{x}+\Lambda_{2}\left[w_{x}+\frac{w}{x}\right]+\Lambda_{3}-D_{x}\left[u \Lambda_{1}+x \Lambda_{3}\right]-D_{y}\left[v \Lambda_{1}\right]-\nu D_{y}^{2}\left(\Lambda_{1}\right)=0 \\
& \Lambda_{1 v}\left(u u_{x}+v u_{y}-\nu u_{y y}-\frac{w^{2}}{x}\right)+\Lambda_{2 v}\left(u w_{x}+v w_{y}+\frac{u w}{x}-\nu w_{y y}\right)+\Lambda_{3 v}\left(u+x u_{x}+x v_{y}\right) \\
& +\Lambda_{1} u_{y}+\Lambda_{2} w_{y}-D_{y}\left(x \Lambda_{3}\right)=0
\end{aligned}
$$

and

$$
\begin{aligned}
& \Lambda_{1 w}\left(u u_{x}+v u_{y}-\nu u_{y y}-\frac{w^{2}}{x}\right)+\Lambda_{2 w}\left(u w_{x}+v w_{y}+\frac{u w}{x}-\nu w_{y y}\right)+\Lambda_{3 w}\left(u+x u_{x}+x v_{y}\right) \\
& -\frac{2 w}{x} \Lambda_{1}+\frac{u}{x} \Lambda_{2}-D_{x}\left[u \Lambda_{2}\right]-D_{y}\left[v \Lambda_{2}\right]-\nu D_{y}^{2}\left(\Lambda_{2}\right)=0
\end{aligned}
$$

Equations (19)-(21) must be satisfied for all functions $u(x, y), v(x, y)$ and $w(x, y)$. After expansion the highest order derivative terms in system (19)-(21) are secondorder derivative terms. Therefore, equating the coefficients of $u_{y y}, v_{y y}$ and $w_{y y}$ to zero, we obtain

$$
\Lambda_{1 u}=0, \Lambda_{1 v}=0, \Lambda_{1 w}=0, \Lambda_{2 u}=0, \Lambda_{2 v}=0, \Lambda_{2 w}=0
$$

which in turn results in

$$
\Lambda_{1}=A(x, y), \Lambda_{2}=B(x, y), \Lambda_{3}=C(x, y, u, v, w)
$$

Equations (19)-(21), with the help of (23), reduce to

$$
\begin{gathered}
x v_{x} C_{v}+v_{y}\left(A-x C_{u}\right)+w_{x}\left(x C_{w}-B\right)-u C_{u}-\frac{B w}{x}+u A_{x}+x C_{x}+v A_{y}+\nu A_{y y}=0 \\
x u_{x} C_{v}+u_{y}\left(A-x C_{u}\right)-w_{y}\left(x C_{w}-B\right)+u C_{v}-x C_{y}=0 \\
\left(u_{x}+v_{y}\right)\left(x C_{w}-B\right)+u C_{w}-\frac{2 w}{x} A+\frac{u}{x} B-u B_{x}-v B_{y}-\nu B_{y y}=0
\end{gathered}
$$

Equating the first-order derivative terms $u_{x}, u_{y}$ and $w_{y}$ in (25) to zero, we obtain

$$
C_{v}=0, A-x C_{u}=0, x C_{w}-B=0,
$$


which yields

$$
C=\frac{u}{x} A(x, y)+\frac{w}{x} B(x, y)+D(x, y) .
$$

Equations (24)-(26) become

$$
\begin{aligned}
& 2 u\left(A_{x}-\frac{A}{x}\right)+v A_{y}+w\left(B_{x}-\frac{2 B}{x}\right)+x D_{x}+\nu A_{y y}=0, \\
& u A_{y}+w B_{y}+x D_{y}=0, \\
& u\left(B_{x}-\frac{2 B}{x}\right)+v B_{y}+\frac{2 w}{x} A+\nu B_{y y}=0 .
\end{aligned}
$$

The separation of equations (29)-(31) with respect to $u, v$ and $w$ finally gives

$$
\Lambda_{1}=0, \Lambda_{2}=c_{2} x^{2}, \Lambda_{3}=c_{1}+c_{2} w x,
$$

where $c_{1}$ and $c_{2}$ are constants. It is not difficult to construct the conserved vectors by elementary manipulations with the help of the multipliers (32). Equations (10) and (32) give

$$
\begin{gathered}
0\left[u u_{x}+v u_{y}-\nu u_{y y}-\frac{w^{2}}{x}\right]+c_{2} x^{2}\left[u w_{x}+v w_{y}+\frac{u w}{x}-\nu w_{y y}\right]+\left(c_{1}+c_{2} w x\right)\left[u+x u_{x}+x v_{y}\right] \\
=D_{x}\left[c_{1} x u+c_{2} x^{2} u w\right]+D_{y}\left[c_{1} x v+c_{2} x^{2}\left(v w-\nu w_{y}\right)\right]
\end{gathered}
$$

for arbitrary functions $u(x, y), v(x, y)$ and $w(x, y)$ and therefore when $u(x, y), v(x, y)$ and $w(x, y)$ are the solutions of the system of differential equations (1)-(3), then

$$
D_{x}\left[c_{1} x u+c_{2} x^{2} u w\right]+D_{y}\left[c_{1} x v+c_{2} x^{2}\left(v w-\nu w_{y}\right)\right]=0 .
$$

Thus

$$
\begin{aligned}
& T^{1}=x u, \quad T^{2}=x v, \\
& T^{1}=x^{2} u w, \quad T^{2}=x^{2}\left(v w-\nu w_{y}\right),
\end{aligned}
$$

are the conserved vectors for the system of differential equations (1)-(3) corresponding to multipliers of the form $\Lambda_{1}=\Lambda_{1}(x, y, u, v, w)$ and $\Lambda_{2}=\Lambda_{2}(x, y, u, v, w)$.

\subsection{Conservation Laws for the stream function formulation}

Consider multipliers of the form $\Lambda_{1}=\Lambda_{1}(x, y, \psi, w)$ and $\Lambda_{2}=\Lambda_{2}(x, y, \psi, w)$ in order to derive the conservation laws for the system (8)-(9). The determining equations for the multipliers $\Lambda_{1}$ and $\Lambda_{2}$ are

$$
E_{\psi}\left[\Lambda_{1}\left(\frac{1}{x} \psi_{y} \psi_{x y}-\frac{1}{x^{2}} \psi_{y}^{2}-\frac{1}{x} \psi_{x} \psi_{y y}-\nu \psi_{y y y}-w^{2}\right)\right.
$$




$$
\left.+\Lambda_{2}\left(\frac{1}{x} \psi_{y} w_{x}-\frac{1}{x} \psi_{x} w_{y}+\frac{1}{x^{2}} \psi_{y} w-\nu w_{y y}\right)\right]=0,
$$

and

$$
\begin{aligned}
& E_{w}\left[\Lambda_{1}\left(\frac{1}{x} \psi_{y} \psi_{x y}-\frac{1}{x^{2}} \psi_{y}^{2}-\frac{1}{x} \psi_{x} \psi_{y y}-\nu \psi_{y y y}-w^{2}\right)\right. \\
& \left.+\Lambda_{2}\left(\frac{1}{x} \psi_{y} w_{x}-\frac{1}{x} \psi_{x} w_{y}+\frac{1}{x^{2}} \psi_{y} w-\nu w_{y y}\right)\right]=0,
\end{aligned}
$$

where

$$
\begin{aligned}
& E_{\psi}=\frac{\partial}{\partial \psi}-D_{x} \frac{\partial}{\partial \psi_{x}}-D_{y} \frac{\partial}{\partial \psi_{y}}+D_{x}^{2} \frac{\partial}{\partial \psi_{x x}}+D_{x} D_{y} \frac{\partial}{\partial \psi_{x y}}+D_{y}^{2} \frac{\partial}{\partial \psi_{y y}}-\cdots \\
& E_{w}=\frac{\partial}{\partial w}-D_{x} \frac{\partial}{\partial w_{x}}-D_{y} \frac{\partial}{\partial w_{y}}+D_{x}^{2} \frac{\partial}{\partial w_{x x}}+D_{x} D_{y} \frac{\partial}{\partial w_{x y}}+D_{y}^{2} \frac{\partial}{\partial w_{y y}}-\cdots
\end{aligned}
$$

are the standard Euler operators. In (39) and (40), $D_{x}$ and $D_{y}$ are the total derivative operators and are defined by

$$
\begin{aligned}
& D_{x}=\frac{\partial}{\partial x}+\psi_{x} \frac{\partial}{\partial \psi}+w_{x} \frac{\partial}{\partial w}+\psi_{x x} \frac{\partial}{\partial \psi_{x}}+w_{x x} \frac{\partial}{\partial w_{x}}+\psi_{x y} \frac{\partial}{\partial \psi_{y}}+w_{x y} \frac{\partial}{\partial w_{y}}+\cdots \\
& D_{y}=\frac{\partial}{\partial y}+\psi_{y} \frac{\partial}{\partial \psi}+w_{y} \frac{\partial}{\partial w}+\psi_{y y} \frac{\partial}{\partial \psi_{y}}+w_{y y} \frac{\partial}{\partial w_{y}}+\psi_{y x} \frac{\partial}{\partial \psi_{x}}+w_{y x} \frac{\partial}{\partial w_{x}}+\cdots .
\end{aligned}
$$

Expansion of equations (37) and (38) yields

$$
\begin{aligned}
& \Lambda_{1 \psi}\left[\frac{1}{x} \psi_{y} \psi_{x y}-\frac{1}{x^{2}} \psi_{y}^{2}-\frac{1}{x} \psi_{x} \psi_{y y}-\nu \psi_{y y y}-w^{2}\right]+\Lambda_{2 \psi}\left[\frac{1}{x} \psi_{y} w_{x}-\frac{1}{x} \psi_{x} w_{y}\right. \\
& \left.+\frac{1}{x^{2}} \psi_{y} w-\nu w_{y y}\right]+D_{x}\left[\frac{1}{x} \Lambda_{1} \psi_{y y}+\frac{1}{x} \Lambda_{2} w_{y}\right]-D_{y}\left[\Lambda_{1}\left(\frac{1}{x} \psi_{x y}-\frac{2}{x^{2}} \psi_{y}\right)\right. \\
& \left.+\Lambda_{2}\left(\frac{w_{x}}{x}+\frac{w}{x^{2}}\right)\right]+D_{x} D_{y}\left[\frac{1}{x} \Lambda_{1} \psi_{y}\right]-D_{y}^{2}\left[\frac{1}{x} \Lambda_{1} \psi_{x}\right]+\nu D_{y}^{3}\left[\Lambda_{1}\right]=0 \\
& \Lambda_{1 w}\left[\frac{1}{x} \psi_{y} \psi_{x y}-\frac{1}{x^{2}} \psi_{y}^{2}-\frac{1}{x} \psi_{x} \psi_{y y}-\nu \psi_{y y y}-w^{2}\right]+\Lambda_{2 w}\left[\frac{1}{x} \psi_{y} w_{x}-\frac{1}{x} \psi_{x} w_{y}+\frac{1}{x^{2}} \psi_{y} w\right. \\
& \left.-\nu w_{y y}\right]-2 w \Lambda_{1}+\frac{\Lambda_{2}}{x^{2}} \psi_{y}-D_{x}\left[\frac{\Lambda_{2}}{x} \psi_{y}\right]+D_{y}\left[\frac{\Lambda_{2}}{x} \psi_{x}\right]-\nu D_{y}^{2}\left[\Lambda_{2}\right]=0
\end{aligned}
$$

Equating the coefficients of $\psi_{y y y}$ and $w_{y y}$ in (44) to zero, we obtain

$$
\Lambda_{1 w}=0, \Lambda_{2 w}=0
$$

Now, since $\Lambda_{1}$ and $\Lambda_{2}$ are not functions of $w$, therefore separating the rest of the terms in (44) (in expanded form) with respect to $w$ yields

$$
\Lambda_{1}=0 \text {. }
$$


Equations (43) and (44) reduce to

$$
\begin{aligned}
& \Lambda_{2 \psi}\left[\frac{1}{x} \psi_{y} w_{x}-\frac{1}{x} \psi_{x} w_{y}+\frac{1}{x^{2}} \psi_{y} w-\nu w_{y y}\right]-2 w_{y} \frac{\Lambda_{2}}{x^{2}}+\frac{w_{y}}{x}\left[\Lambda_{2 x}+\psi_{x} \Lambda_{2 \psi}\right] \\
& -\left(\frac{w_{x}}{x}+\frac{w}{x^{2}}\right)\left[\Lambda_{2 y}+\psi_{y} \Lambda_{2 \psi}\right]=0,
\end{aligned}
$$

and

$$
\begin{aligned}
& 2 \psi_{y} \frac{\Lambda_{2}}{x^{2}}-\frac{\psi_{y}}{x}\left[\Lambda_{2 x}+\psi_{x} \Lambda_{2 \psi}\right]+\frac{\psi_{x}}{x}\left[\Lambda_{2 y}+\psi_{y} \Lambda_{2 \psi}\right] \\
& -\nu\left[\Lambda_{2 y y}+\psi_{y} \Lambda_{2 \psi y}+\psi_{y y} \Lambda_{2 \psi}+\psi_{y} \Lambda_{2 \psi y}+\psi_{y}^{2} \Lambda_{2 \psi \psi}\right]=0 .
\end{aligned}
$$

From equation (47), the coefficient of $w_{y y}$ (or in (48) the coefficient of $\psi_{y y}$ ) yields $\Lambda_{2 \psi}=0$ and thus

$$
\Lambda_{2}=A(x, y)
$$

Equations (47) and (48) become

$$
\begin{aligned}
& w_{x} \frac{A_{y}}{x}+w_{y}\left[\frac{2 A}{x^{2}}-\frac{A_{x}}{x}\right]+w \frac{A_{y}}{x^{2}}=0, \\
& \psi_{x} \frac{A_{y}}{x}+\psi_{y}\left[\frac{2 A}{x^{2}}-\frac{A_{x}}{x}\right]-\nu A_{y y}=0,
\end{aligned}
$$

which finally yield $A=c_{3} x^{2}$ and

$$
\Lambda_{1}=0, \Lambda_{2}=c_{3} x^{2}
$$

where $c_{3}$ is an arbitrary constant.

The multipliers $\Lambda_{1}$ and $\Lambda_{2}$ for the system (8)-(9) satisfy

$$
\begin{aligned}
& \Lambda_{1}\left[\frac{1}{x} \psi_{y} \psi_{x y}-\frac{1}{x^{2}} \psi_{y}^{2}-\frac{1}{x} \psi_{x} \psi_{y y}-\nu \psi_{y y y}-w^{2}\right] \\
& +\Lambda_{2}\left[\frac{1}{x} \psi_{y} w_{x}-\frac{1}{x} \psi_{x} w_{y}+\frac{1}{x^{2}} \psi_{y} w-\nu w_{y y}\right] \\
& =D_{x} T^{1}+D_{y} T^{2}
\end{aligned}
$$

for all functions $\psi(x, y)$ and $w(x, y)$. From equations (52) and (53), we have

$$
\begin{aligned}
& 0\left[\frac{1}{x} \psi_{y} \psi_{x y}-\frac{1}{x^{2}} \psi_{y}^{2}-\frac{1}{x} \psi_{x} \psi_{y y}-\nu \psi_{y y y}-w^{2}\right]+c_{3} x^{2}\left[\frac{1}{x} \psi_{y} w_{x}-\frac{1}{x} \psi_{x} w_{y}+\frac{1}{x^{2}} \psi_{y} w-\nu w_{y y}\right] \\
& \quad=D_{x}\left[c_{3} x w \psi_{y}\right]+D_{y}\left[c_{3}\left(-x w \psi_{x}-\nu x^{2} w_{y}\right)\right]
\end{aligned}
$$


for arbitrary $\psi(x, y)$ and $w(x, y)$. When $\psi(x, y)$ and $w(x, y)$ are solutions of the system (8)-(9), we have

$$
D_{x}\left[c_{3} x w \psi_{y}\right]+D_{y}\left[c_{3}\left(-x w \psi_{x}-\nu x^{2} w_{y}\right)\right]=0 .
$$

Thus

$$
T^{1}=x w \psi_{y}, T^{2}=-x w \psi_{x}-\nu x^{2} w_{y}
$$

are the components of a conserved vector for the system (8)-(9). Notice that the conserved vector (56) is equivalent to the conserved vector (36).

The multiplier approach gives multipliers only for the local conserved vectors. The multipliers for the non-local conserved vectors cannot be obtained with the help of the multiplier approach as presented. Notice that if we multiply equation (8) with zero and equations (9) with $x^{2} \psi$, we have

$$
\begin{aligned}
& 0\left[\frac{1}{x} \psi_{y} \psi_{x y}-\frac{1}{x^{2}} \psi_{y}^{2}-\frac{1}{x} \psi_{x} \psi_{y y}-\nu \psi_{y y y}-w^{2}\right]+x^{2} \psi\left[\frac{1}{x} \psi_{y} w_{x}-\frac{1}{x} \psi_{x} w_{y}+\frac{1}{x^{2}} \psi_{y} w-\nu w_{y y}\right] \\
& \quad=D_{x}\left[x \psi w \psi_{y}\right]+D_{y}\left[-x w \psi \psi_{x}-\nu x^{2} \psi w_{y}+\nu x^{2} \int^{y} w_{y} \psi_{y} d y\right]
\end{aligned}
$$

for arbitrary $\psi(x, y)$ and $w(x, y)$. When $\psi(x, y)$ and $w(x, y)$ are solutions of the system (8)-(9), we have

$$
D_{x}\left[x \psi w \psi_{y}\right]+D_{y}\left[-x w \psi \psi_{x}-\nu x^{2} \psi w_{y}+\nu x^{2} \int^{y} w_{y} \psi_{y} d y\right]=0
$$

Thus

$$
T^{1}=x \psi w \psi_{y}, T^{2}=-x w \psi \psi_{x}-\nu x^{2} \psi w_{y}+\nu x^{2} \int^{y} w_{y} \psi_{y} d y
$$

are the components of a non-local conserved vector for the system (8)-(9). It may be possible that the non-local conservation law (59) which does not arise from local multipliers can be derived through a potential system associated with the system (1), (2) and (3) or the system (8) and (9).

\subsection{Conservation laws for radial jets at large distance from the orifice}

The radial jets with swirl at large distance from the orifice, correspond to a radial jet with zero swirl. When $x \rightarrow \infty, w \rightarrow 0$, then the system (1)-(3) reduces to

$$
\begin{aligned}
& u u_{x}+v u_{y}=\nu u_{y y}, \\
& (x u)_{x}+(x v)_{y}=0,
\end{aligned}
$$


which is same as the boundary layer equations for purely radial jet flows. The system (8)-(9) for the stream function, when $x \rightarrow \infty$, reduces to a single third-order partial differential equation

$$
\frac{1}{x} \psi_{y} \psi_{x y}-\frac{1}{x^{2}} \psi_{y}^{2}-\frac{1}{x} \psi_{x} \psi_{y y}-\nu \psi_{y y y}=0
$$

The local conserved vectors for system (60)-(61) derived in [7] are

$$
\begin{aligned}
& T^{1}=x u, \quad T^{2}=x v, \\
& T^{1}=x u^{2}, \quad T^{2}=x u v-\nu x u_{y} .
\end{aligned}
$$

The conserved vectors for the third-order partial differential equation (62) (see [7]) are

$$
T^{1}=\frac{1}{x} \psi_{y}^{2}, T^{2}=-\frac{1}{x} \psi_{x} \psi_{y}-\nu \psi_{y y}
$$

and

$$
T^{1}=\frac{1}{x} \psi \psi_{y}^{2}, T^{2}=-\frac{1}{x} \psi \psi_{x} \psi_{y}+\frac{\nu}{2} \psi_{y}^{2}-\nu \psi \psi_{y y} .
$$

The conserved vectors given in equations (64) and (65) are equivalent.

\section{CONSERVED QUANTITIES FOR RADIAL LIQUID JETS}

In this section, we derive the conserved quantities for the radial liquid, free and wall jets with swirl by the technique used in [7]. We first consider the conserved vectors (35), (36) and (64) for the system of equations for the velocity components and derive the conserved quantities for the radial liquid and the free jets with swirl. Then we consider the stream function formulation to give an alternative derivation of the conserved quantities for the radial free jet with swirl and also derive the conserved quantities for the radial wall jet with swirl.

All the conserved vectors $\left(T^{1}, T^{2}\right)$ derived here depend on $u(x, y), v(x, y)$, $w(x, y)$ or $\psi(x, y)$ and satisfy

$$
D_{x} T^{1}+D_{y} T^{2}=\frac{\partial T^{1}}{\partial x}+\frac{\partial T^{2}}{\partial y} .
$$

But for a conserved vector $D_{x} T^{1}+D_{y} T^{2}=0$ and therefore equation (67) yields

$$
\frac{\partial T^{1}}{\partial x}+\frac{\partial T^{2}}{\partial y}=0
$$


The conserved quantities for the radial liquid, free and wall jets with swirl are obtained by integrating equation (68) for the corresponding conservation law across the jet and imposing the boundary conditions.

\subsection{Conserved quantity for the radial liquid jet with swirl}

The conserved quantity for the radial liquid jet with swirl is derived with the help of conserved vector (35). The derivation for conserved quantity for radial liquid jet with swirl is same as in [7] for the purely radial jet flow. The boundary conditions for the radial liquid jet with swirl are

$$
\begin{aligned}
& y=0: \quad u=0, v=0, w=0, \\
& y=\phi(x): \quad u_{y}=0, w_{y}=0 .
\end{aligned}
$$

The velocity component $v(x, \phi(x))$ is

$$
v(x, \phi(x))=\frac{D}{D t}[\phi(x)]=u(x, \phi(x)) \frac{d \phi(x)}{d x},
$$

where

$$
\frac{D}{D t}=\frac{\partial}{\partial t}+u(x, y) \frac{\partial}{\partial x}+v(x, y) \frac{\partial}{\partial y}
$$

is the material time derivative. The conserved quantity for the radial liquid jet with swirl is obtained by integrating (68) with respect to $y$ from $y=0$ to $y=\phi(x)$ keeping $x$ as fixed. For the conserved vector (35), we obtain

$$
\int_{0}^{\phi(x)}\left[\frac{\partial}{\partial x}(x u)+\frac{\partial}{\partial y}(x v)\right] d y=0 .
$$

Differentiating (73) under integration sign [12], we have

$$
\frac{d}{d x} \int_{0}^{\phi(x)}[x u] d y-x u(x, \phi(x)) \frac{d \phi(x)}{d x}+[x v(x, y)]_{0}^{\phi(x)}=0 .
$$

The boundary condition (69) for $v(x, 0)$ and expression (71) for $v(x, \phi(x))$, reduces (74) to

$$
x \int_{0}^{\phi(x)} u d y=\text { constant, independent of } x,
$$

which gives that the total mass flux is constant along the jet. Therefore

$$
F=x \int_{0}^{\phi(x)} u d y
$$


is the conserved quantity for the radial liquid jet with swirl [5].

\subsection{Conserved quantities for the radial free jet with swirl}

The boundary conditions for the radial free jet with swirl are

$$
\begin{aligned}
& y=0: \quad v=0, u_{y}=0, w_{y}=0, \\
& y= \pm \infty: u=0, u_{y}=0, w=0, w_{y}=0 .
\end{aligned}
$$

For the conserved vector (36), equation (68) on integration with respect to $y$ from $y=-\infty$ to $y=\infty$ with $x$ kept as fixed during the integration, yields

$$
\frac{d}{d x}\left[x^{2} \int_{-\infty}^{\infty}(u w) d y\right]+\left[x^{2} v w-\nu x^{2} w_{y}\right]_{-\infty}^{\infty}=0
$$

We assume that $v(x, \pm \infty)$ is finite. The boundary conditions (77) and (78) on (79) gives

$$
2 x^{2} \int_{0}^{\infty} u w d y=\text { constant, independent of } x .
$$

The conserved quantity for the radial free jet with swirl is

$$
F=2 x^{2} \int_{0}^{\infty} u w d y
$$

where $F$ is a constant independent of $x$. If $\rho$ is the density of fluid, then the constant $\rho F$ is the total radial flux of angular momentum about $x=0$ (Riley [5]). The second conserved quantity which is valid only when there is no swirl or at large distance from the orifice can be obtained from the conserved vector (64). Integrate (68) with respect to $y$ for the conserved vector (64), we obtain

$$
\frac{d}{d x}\left[x \int_{-\infty}^{\infty} u^{2} d y\right]+\left[x u v-\nu x u_{y}\right]_{-\infty}^{\infty}=0
$$

The boundary condition (78) and $v(x, \pm \infty)$ is finite, yields

$$
2 x \int_{0}^{\infty} u^{2} d y=\text { constant, independent of } x .
$$

Thus

$$
G=2 x \int_{0}^{\infty} u^{2} d y
$$

and $\rho G$ is the second conserved quantity for the radial free jet with swirl and is valid only at large distance from orifice. 
The conserved quantities for the radial free jet with swirl can also be derived for the stream function formulation. We now outline an alternative derivation of the conserved quantities for the radial free jet with swirl. Also we derive the conserved quantities for the radial wall jet with swirl.

In terms of the stream function the boundary conditions (77) and (78) take the following form:

$$
\begin{aligned}
& y=0: \quad \psi_{x}=0, \psi_{y y}=0, w_{y}=0 \\
& y= \pm \infty: \quad \psi_{y}=0, \psi_{y y}=0, w=0, w_{y}=0 .
\end{aligned}
$$

Integrate equation (68) with respect to $y$ form $y=-\infty$ to $y=\infty$ keeping $x$ as fixed. For the conserved vector (56), we have

$$
\frac{d}{d x}\left[x \int_{-\infty}^{\infty} w \psi_{y} d y\right]+\left[-x w \psi_{x}-\nu x^{2} w_{y}\right]_{-\infty}^{\infty}=0
$$

Equation (87) with the help of boundary condition (86) and requirement $\psi_{x}(x, \pm \infty)=$ $-x v(x, \pm \infty)$ is finite, gives

$$
2 x \int_{0}^{\infty} w \psi_{y} d y=\text { constant, independent of } x .
$$

Equation (88) is equivalent to (80) and thus we finally obtain the conserved quantity $F$ given in (81). The second conserved quantity which is valid only when there is no swirl or at large distance from the orifice can be obtained from conserved vector (65). Integration of (68) with respect to $y$ with $x$ kept as fixed for the conserved vector (65) gives

$$
\frac{d}{d x}\left[\frac{1}{x} \int_{-\infty}^{\infty} \psi_{y}^{2} d y\right]+\left[-\frac{1}{x} \psi_{x} \psi_{y}-\nu \psi_{y y}\right]_{-\infty}^{\infty}=0
$$

Equation (89) with the help of boundary condition (86) and the requirement $\psi_{x}(x, \pm \infty)=$ $-x v(x, \pm \infty)$ is finite, yields

$$
\frac{2}{x} \int_{0}^{\infty} \psi_{y}^{2} d y=\text { constant, independent of } x
$$

Equation (90) is equivalent to (83) and hence we obtain the conserved quantity (84).

\subsection{Conserved quantities for the radial wall jet with swirl}

For the radial wall jet with swirl the boundary conditions are

$$
y=0: \quad u=0, v=0, w=0,
$$




$$
y=\infty: \quad u=0, u_{y}=0, w=0, w_{y}=0 .
$$

In terms of the stream function, we obtain

$$
\begin{aligned}
& y=0: \quad \psi_{x}=0, \psi_{y}=0, w=0, \\
& y=\infty: \quad \psi_{y}=0, \psi_{y y}=0, w=0, w_{y}=0 .
\end{aligned}
$$

By integrating equation (68) with respect to $y$ from $y=0$ to $y=\infty$ keeping $x$ as fixed and considering the conserved vector (59), we have

$$
\frac{d}{d x}\left[x \int_{0}^{\infty} \psi w \psi_{y} d y\right]+\left[-x w \psi \psi_{x}-\nu x^{2} \psi w_{y}+\nu x^{2} \int^{y} w_{y} \psi_{y} d y\right]_{0}^{\infty}=0 .
$$

But $\psi(x, 0)=0$ whereas $\psi(x, \infty), \psi_{x}(x, \infty)$ and $w_{y}(x, 0)$ are assumed to be finite. Thus boundary conditions (93) and (94) reduce equation (95) to

$$
\frac{d}{d x}\left[x \int_{0}^{\infty} \psi w \psi_{y} d y\right]+\nu x^{2} \int_{0}^{\infty} w_{y} \psi_{y} d y=0 .
$$

Integrating equation (96) by parts and using boundary conditions, we deduce

$$
\frac{d}{d x}\left(x \int_{0}^{\infty} \psi_{y}\left(\int_{y}^{\infty} w \psi_{y^{*}} d y^{*}\right) d y\right)=\nu x^{2} \int_{0}^{\infty} w \psi_{y y} d y
$$

and in terms of the velocity components, we have

$$
\frac{d}{d x}\left(x^{3} \int_{0}^{\infty} u\left(\int_{y}^{\infty} w u d y^{*}\right) d y\right)=\nu x^{3} \int_{0}^{\infty} w u_{y} d y
$$

To make the right side of equation (98) zero, Riley [5] assumed that $u / w$ is a function of $x$ only and this condition is satisfied by the similarity solution. Thus in the similarity solution regime, we have

$$
x^{3} \int_{0}^{\infty} u\left(\int_{y}^{\infty} w u d y^{*}\right) d y=\text { constant, independent of } x .
$$

Therefore

$$
M=x^{3} \int_{0}^{\infty} u\left(\int_{y}^{\infty} w u d y^{*}\right) d y
$$

is a conserved quantity. Riley [5] used $\rho^{2} M$ as a conserved quantity for the radial wall jet with swirl. There is no obvious physical significance of this conserved quantity.

The second conserved quantity is derived with no swirl or at large distance from the orifice and the conserved vector (66) is used in its derivation. Integrating (68) 
with respect to $y$ from $y=0$ to $y=\infty$ keeping $x$ as fixed during the integration, we have

$$
\frac{d}{d x}\left[\frac{1}{x} \int_{0}^{\infty} \psi \psi_{y}^{2} d y\right]+\left[-\frac{1}{x} \psi \psi_{x} \psi_{y}+\frac{\nu}{2} \psi_{y}^{2}-\nu \psi \psi_{y y}\right]_{0}^{\infty}=0 .
$$

The boundary conditions (93) and (94) yield

$$
\frac{1}{x} \int_{0}^{\infty} \psi \psi_{y}^{2} d y=\text { constant, independent of } x
$$

we have used the requirements $\psi(x, 0)=0, \psi(x, \infty)$ and $\psi_{x}(x, \infty)$ are finite. Integrating (102) by parts, we obtain

$$
N=\frac{1}{x} \int_{0}^{\infty} \psi_{y}\left(\int_{y}^{\infty} \psi_{y^{*}}^{2} d y^{*}\right) d y
$$

or equivalently

$$
N=x^{2} \int_{0}^{\infty} u\left(\int_{y}^{\infty} u^{2} d y^{*}\right) d y
$$

which is valid only for no swirl or at large distance from orifice. The constant $\rho^{2} N$ is interpreted as the flux of exterior momentum flux.

\subsection{Comparison between conservation laws for radial jets with swirl and purely radial jet flows}

The results for radial jets with swirl and for purely radial jets derived in [7] are displayed in Table 1 . The boundary layer equations for the velocity components as well as for the stream function formulation are given in Table 1 for both the radial jets with swirl and for the purely radial jets. The conservation laws for the purely radial jet flows hold for the radial jets with swirl but only at large distances from the orifice (as $x \rightarrow \infty$ ). The multiplier approach on the system of equations for the velocity components for purely radial jets yields two local conserved vectors and for the stream function formulation also two local conserved vectors are obtained. The multiplier approach on the system of equations for the velocity components for radial jets with swirl yields three conserved vectors, one of these conserved vectors is valid only when $x \rightarrow \infty$. For the stream function formulation for the radial jets with swirl four conserved vectors are obtained and two of them are valid only when $x \rightarrow \infty$. The conserved vectors for the radial jets with swirl as $x \rightarrow \infty$ and for the purely radial jets are the same.

The conserved quantity for the radial liquid jet with swirl and the purely radial liquid jet are the same. The only conserved quantity for the purely radial free jet 
is that the total radial flux of momentum is constant in the jet. The radial free jet with swirl has two conserved quantities. One conserved quantity for the radial free jet with swirl is the total radial flux of angular momentum about $x=0$ which is constant. The second conserved quantity is the same as for the purely radial free jet but it holds only as $x \rightarrow \infty$. The conserved quantity for a purely radial wall jet is the flux of exterior momentum flux in the wall jet which is constant and holds for the radial wall jet with swirl at $x \rightarrow \infty$. Another conserved quantity for the radial wall jet with swirl is established with the help of a non-local conserved vector for the system of two partial differential equations for the stream function formulation. There is no obvious physical interpretation of that conserved quantity. 


\section{Radial jets with swirl $\quad$ Purely radial jets}

Boundary layer

$u u_{x}+v u_{y}-\frac{w^{2}}{x}=\nu u_{y y}$
$u u_{x}+v u_{y}=\nu u_{y y}$ equations

$$
\begin{array}{rlrl}
u w_{x}+v w_{y}+\frac{u w}{x} & =\nu w_{y y} & & \\
(x u)_{x}+(x v)_{y} & =0 & (x u)_{x}+(x v)_{y}=0
\end{array}
$$

Conserved vectors $\quad T^{1}=x^{2} u w$

for

$$
T^{2}=x^{2}\left(v w-\nu w_{y}\right)
$$

velocity components

$$
\begin{array}{ll}
T^{1}=x u & T^{1}=x u \\
T^{2}=x v & T^{2}=x v
\end{array}
$$

As $x \rightarrow \infty$

$$
\begin{aligned}
& T^{1}=x u^{2} \\
& T^{2}=x\left(u v-\nu u_{y}\right)
\end{aligned}
$$$$
T^{1}=x u^{2}
$$$$
T^{2}=x\left(u v-\nu u_{y}\right)
$$

Stream function

$$
\begin{gathered}
\frac{1}{x} \psi_{y} \psi_{x y}-\frac{1}{x^{2}} \psi_{y}^{2}-\frac{1}{x} \psi_{x} \psi_{y y} \\
-\nu \psi_{y y y}-w^{2}=0 \\
\frac{1}{x} \psi_{y} w_{x}-\frac{1}{x} \psi_{x} w_{y}+\frac{1}{x^{2}} \psi_{y} w \\
-\nu w_{y y}=0
\end{gathered}
$$$$
\frac{1}{x} \psi_{y} \psi_{x y}-\frac{1}{x^{2}} \psi_{y}^{2}
$$

formulation

$$
\begin{aligned}
& \text { Conserved vectors } \quad T^{1}=x w \psi_{y} \\
& \text { for stream function } \quad T^{2}=-x w \psi_{x}-\nu x^{2} w_{y} \\
& T^{1}=x \psi w \psi_{y} \\
& T^{2}=-x w \psi \psi_{x}-\nu x^{2} \psi w_{y} \\
& +\int_{0}^{y} \nu x^{2} w_{y} \psi_{y} \\
& \text { As } x \rightarrow \infty \\
& T^{1}=\frac{1}{x} \psi_{y}^{2} \quad T^{1}=\frac{1}{x} \psi_{y}^{2} \\
& T^{2}=-\frac{1}{x} \psi_{x} \psi_{y}-\nu \psi_{y y} \\
& T^{1}=\frac{1}{x} \psi \psi_{y}^{2} \\
& T^{2}=-\frac{1}{x} \psi \psi_{x} \psi_{y}+\frac{\nu}{2} \psi_{y}^{2} \\
& T^{2}=-\frac{1}{x} \psi_{x} \psi_{y}-\nu \psi_{y y} \\
& T^{1}=\frac{1}{x} \psi \psi_{y}^{2} \\
& T^{2}=-\frac{1}{x} \psi \psi_{x} \psi_{y}+\frac{\nu}{2} \psi_{y}^{2} \\
& -\nu \psi \psi_{y y} \\
& -\nu \psi \psi_{y y}
\end{aligned}
$$


Conserved quantities

For liquid jet

$x \int_{0}^{\phi(x)} u d y$

$x \int_{0}^{\phi(x)} u d y$

For free jet

$$
2 x^{2} \int_{0}^{\infty} u w d y
$$

$$
2 x \int_{0}^{\infty} u^{2} d y \text { as } x \rightarrow \infty \quad 2 x \int_{0}^{\infty} u^{2} d y
$$

For wall jet

$$
\begin{aligned}
& x^{3} \int_{0}^{\infty} u\left(\int_{y}^{\infty} w u d y^{*}\right) d y \\
& x^{2} \int_{0}^{\infty} u\left(\int_{y}^{\infty} u^{2} d y^{*}\right) d y \quad x^{2} \int_{0}^{\infty} u\left(\int_{y}^{\infty} u^{2} d y^{*}\right) d y \\
& \quad \text { as } x \rightarrow \infty
\end{aligned}
$$

Table 1: Comparison between radial jets with swirl and purely radial jets

\section{CONCLUDING REMARKS}

The conserved quantities for the radial liquid, free and wall jets with swirl derived by Riley [5] can be constructed with the help of conservation laws. The radial jets with swirl behave as purely radial jets at large distances from the orifice. At large distances from the orifice as $x \rightarrow \infty$, the swirling component $w \rightarrow 0$ and the boundary layer equations for radial jets with swirl reduce to that of purely radial jets. Therefore, as $x \rightarrow \infty$ the conservation laws for radial jets with swirl coincide with those of the purely radial jets.

The multiplier approach gave two local conservation laws for the system of equations for the velocity components. One of the conserved vectors gave the conserved quantity for the radial liquid jet with swirl and the second conserved vector yielded one conserved quantity for the radial free jet with swirl. Also, another conserved vector for this system was obtained as $x \rightarrow \infty$. The second conserved quantity for the radial free jet with swirl was obtained from that conserved vector. The conserved quantities for the wall jet with swirl cannot be obtained from the conserved vectors for the system of equations for the velocity components.

For the system of two partial differential equations for the stream function formulation one local and one non-local conserved vector were obtained. The local conserved vector gave one conserved quantity for the radial free jet with swirl and the non-local conserved vector was used to establish one conserved quantity for the radial wall jet with swirl. Also, two conserved vectors for the stream function for- 
mulation were obtained as $x \rightarrow \infty$ which were used to derive the second conserved quantities for the radial free and wall jets with swirl.

It is of interest to notice that the non-local conserved vector for the system of two partial differential equations for the stream function formulation cannot be obtained by the multiplier approach. The reason is that the multiplier approach only gives multipliers for the local conserved vectors. It may be possible that the non-local conservation law which does not arise from local multipliers can be derived through a potential system associated with the system (1)-(3) or the system (8)-(9).

\section{REFERENCES}

[1] E. J. Watson, The radial spread of a liquid jet over a horizontal plane, J. Fluid Mech, 20, 481-499, 1964.

[2] W. H. Schwarz, The radial free jet, Chem. Eng, Sci, 18, 779-786, 1963.

[3] M. B. Glauert, The wall jet, J. Fluid Mech,1, 625-643, 1956.

[4] N. Riley, Asymptotic expansions in radial jets, J. Math. Phys, 41, 132-146, 1962.

[5] N. Riley, Radial jets with swirl, Part I. Incompressible Flow, Quart. J. Mech. and Appl. Math, 15, 435-458, 1962.

[6] M. O'Nan , W. H. Schwarz, The swirling radial free jet, Appl. Sci. Res. 15, 289-312, 1966.

[7] R. Naz, D. P. Mason and F. M. Mahomed, Conservation laws and conserved quantities for laminar two-dimensional and radial jets, Nonlinear Analysis: Real World Applications, 10, 2641-2651, 2009.

[8] H. Steudel, Uber die Zuordnung Zwischen Invarianzeigenschaften und Erhaltungssatzen, Zeit Naturforsch, 17, 129-132, 1962.

[9] P. J. Olver, Applications of Lie Groups to Differential Equations, Springer, New York, 1993.

[10] R. Naz, F. M. Mahomed, D. P. Mason, Comparison of different approaches to conservation laws for some partial differential equations in fluid mechanics, Appl. Math.Comp, 205, 212-230, 2008.

[11] G. K. Batchelor, An Introduction to Fluid Dynamics, Cambridge University Press, 75-79, 1967.

[12] R. P. Gillespie, Integration, Oliver and Boyd, Edinburgh, 113-116, 1967. 